\title{
Geometry dependence of the conductance fluctuations in metallic nanostructures
}

\author{
E. Scheer ${ }^{a}{ }^{*}$, H.v. Löhneysen ${ }^{a}$, H. Hein ${ }^{b}$ \\ a Physikalisches Institut, Universität Karlsruhe, Engesserstraße 7, D-76128 Karlsruhe, Germany \\ ${ }^{\mathrm{b}}$ Forschungszentrum Karlsruhe, Institut für Mikrostrukturtechnik, D-76021 Karlsruhe, Germany
}

\begin{abstract}
We investigate fluctuations in the magnetoconductance of noble-metal nanostructures in a mesoscopic two-lead configuration. The samples are gold and silver wires with lengths 500 and $1000 \mathrm{~nm}$ and widths $w$ between 45 and $340 \mathrm{~nm}$. The correlation field $B_{c}$ varies as $w^{-1}$. Furthermore, the angle $\theta$ between the direction of the magnetic field and the current is changed continuously from $90^{\circ}$ (usual geometry) to $0^{\circ}$. The dependence of $B_{\mathrm{c}}$ on $\theta$ is weaker than $B_{\mathrm{c}} \propto B_{\mathrm{c} \perp} / \sin \theta$.
\end{abstract}

\section{Introduction}

Quantum interference effects in metals, e.g., aperiodic fluctuations of the magnetoconductance of the order of a universal value $e^{2} / h[1]$ or periodic oscillations of the magnetoconductance of ring structures [2], can be observed if the sample dimensions are smaller than or comparable to the phase coherence length $l_{\phi}=\left(D \tau_{\phi}\right)^{1 / 2}\left(D=v_{\mathrm{F}} l / 3\right.$ is the diffusion constant and $\tau_{\phi}$ is the phase breaking time) of the electrons diffusing through the sample. In polycrystalline metal films $l_{\phi}$ is about $1 \mu \mathrm{m}$ at very low temperatures $T<1 \mathrm{~K}$, whereas the elastic mean free path $l$ is about $10-50 \mathrm{~nm}$. In this diffusive regime the electrical conductance $G$ is influenced by the interference of paths which an electron can travel along its way through the sample, i.e. $G$ fluctuates around a mean value. The RMS-amplitude of the fluctuations depends only very weakly on the sample shape (length $L$, width $w$, and thickness $d$ ) as long as the transport is diffusive and coherent $\left(l \ll w, d, L<l_{\phi}\right)$ [3]. The amplitude of the universal conductance fluctuations (UCF) is determined by the correlation function of the conductances over an ensemble of different samples with the same macroscopic dimensions and mean value of $G$ but varying positions of scattering centers. The ensemble can be obtained experimentally by altering the configuration of the scattering centers [4], by chang-

* Corresponding author. ing the Fermi energy [5], the transport voltage [6,7], or the magnetic field [1]. The correlation functions for all these realizations are essentially the same with the only exception that the RMS-amplitude as a function of the magnetic field $B$ is smaller by a factor $\sqrt{2}$ because of time-reversal symmetry breaking [3]. An additional reduction by a factor of 2 occurs in metals with strong spinorbit scattering. Altogether, one expects a RMS-amplitude of magnetoconductance fluctuations for $\mathrm{Au}$ or $\mathrm{Ag}$ of $\delta G_{1 \mathrm{D}}=0.26 e^{2} / h$ for wires and $\delta G_{2 \mathrm{D}}=0.31 e^{2} / h$ for twodimensional films.

Experiments on long one-dimensional gold wires with two wide probes $[8]$ and silver wires with two wide current probes and two narrow voltage probes [9] show $\delta G \approx$ $0.12 e^{2} / h$. The reduction is attributed to the reduced backscattering probability from the wide probes into the narrow wires and is in qualitative agreement with the $a / l$ reduction of the UCF amplitude in quasi-ballistic point contacts with diameter $a[6,7]$.

The characteristic fluctuation period $B_{\mathrm{c}}$, the correlation field, is a measure of the area $A_{\phi}$ surrounded by typical interference paths since $A_{\phi} B_{\mathrm{c}}=C \phi_{0}$ with the elementary flux quantum $\phi_{0}=h / e$. Here $C$ is a constant of order $1[3,13]$. Because all coherent paths contribute to the interference pattern, $B_{\mathrm{c}}$ should depend on the geometry of the sample if the width and/or the length of the sample become smaller than $l_{\phi}$. At finite temperature $l_{\phi}$ decreases mainly because of the increase of inelastic scattering events. In addition, a 
thermal smearing occurs which can be accounted for by the thermal diffusion length $l_{\mathrm{T}}=\left(h D / k_{\mathrm{B}} T\right)^{1 / 2}$ [3]. In analogy, the energy range $e V_{\mathrm{m}}$ ( $V_{\mathrm{m}}$ is the measuring $\mathrm{AC}$ voltage) generates the Thouless length scale $l_{\mathrm{V}}=\left(h D / e V_{\mathrm{m}}\right)^{1 / 2}$. For $w, d<l_{\phi}<L$ one expects that the correlation field is determined by the width $w$ of the sample and $l_{\phi}$ if the sample length is smaller than $\min \left(l_{\phi}, l_{\mathrm{T}}, l_{\mathrm{V}}\right)$. We will report on measurements of the magnetoconductance, the so-called "magnetofingerprints", of gold and silver wires in a two-lead configuration with wide funnel-shaped leads, with lengths $L \geqslant 500 \mathrm{~nm}$ and widths varying between 45 and $340 \mathrm{~nm}$ at $T \lesssim 100 \mathrm{mK}\left(l_{\phi} \leqslant 2 \mu \mathrm{m}\right)$. We also report on an experiment where the effective geometry is changed by tilting the sample with respect to the field direction.

\section{Experimental}

The samples are produced by a standard lift-off process with a bilayer resist and thermal evaporation of the metal film to a thickness of about $20-30 \mathrm{~nm}$ [10]. The mesoscopic samples and the contact leads are prepared in a single evaporation step and therefore have the same sheet resistance $R_{\square}$. The measurements are performed within the mixing chamber made of araldite of a ${ }^{3} \mathrm{He} /{ }^{4} \mathrm{He}$-dilution refrigerator which is equipped with a $13 \mathrm{~T}$ superconducting solenoid. An ACresistance bridge is used to record the fluctuations with a voltage resolution of about $1 \mathrm{nV}$. The measuring temperature was always below $100 \mathrm{mK}$. A mechanical feedthrough allows to turn the sample while it is maintained at low temperatures continuously from the usual geometry with $B$ perpendicular to the film plane $\left(\theta=90^{\circ}\right)$ to a parallel alignment between $B$ and current direction.

\section{Results and discussion}

The resistivity of the samples is $\rho \approx 3-5 \mu \Omega \mathrm{cm}$ and the sheet resistance $R_{\square}$ lies between 1 and $3 \Omega$. The elastic mean free path of the electrons is deduced from the residual resistance ratio $R R R=R(T=300 \mathrm{~K}) / R(T \rightarrow 0) \approx$ $1+l / l_{\mathrm{ph}}(300 \mathrm{~K})$ with the known electron-phonon scattering lengths $l_{\mathrm{ph}}$ of gold and silver at $300 \mathrm{~K}$ to about $25-30 \mathrm{~nm}$. Due to the two-lead geometry one measures the resistance $R_{\mathrm{c}}$ of the contact leads in series with the resistance of the mesoscopic sample. $R_{\mathrm{c}}$ is calculated to be about $5-7 R_{\square}$ depending on the angle of the funnels. For the wider samples ( $w>200 \mathrm{~nm}$ ), $R_{\mathrm{c}}$ exceeds the sample resistance $R_{\mathrm{s}}$ itself. We scale the measured resistance fluctuations $\delta R(B)$ by subtracting $R_{\mathrm{c}}$ from the total resistance $R_{\mathrm{s}}=R-R_{\mathrm{c}}$, to obtain the conductance fluctuations

$$
\delta G(B)=-\frac{\delta R(B)}{R_{\mathrm{s}}^{2}},
$$

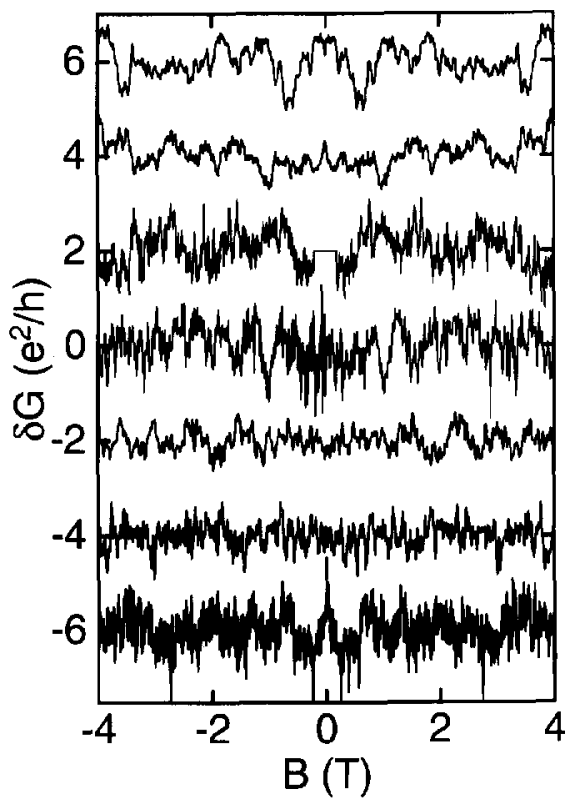

Fig. 1. Conductance fluctuations for wires of length $500 \mathrm{~nm}$ and varying width $w$, offset by $2 e^{2} / h$ each. From top to bottom $w=45,80,100,120,160,240$, and $320 \mathrm{~nm}$.

i.e. assuming non-fluctuating contact leads. The route of the numerical data analysis has been explained in detail elsewhere [10].

Fig. 1 shows $\delta G(B)$ of various wires with $L=500 \mathrm{~nm}$ and varying $w$ for the field range $-4 \mathrm{~T} \leqslant B \leqslant 4 \mathrm{~T}$. The fluctuation amplitude is almost the same for all samples, $\operatorname{RMS}(G)=$ $(0.26 \pm 0.07) e^{2} / h$. This means that the assumption of nonfluctuating contact leads is almost fulfilled. Scaling $\delta R(B)$ to the total resistance would lead to a variance of $\operatorname{RMS}(G)$ of more than two orders of magnitude which would be hard to understand for this ensemble of similarly prepared samples. The calculated RMS-amplitudes are in good agreement with the theoretical predictions. Within our accuracy we cannot distinguish between 1D and 2D behavior. To our knowledge, the predicted amplitude of $\approx 0.25-0.31 e^{2} / h$ has not been observed in gold or silver samples in a two-lead configuration up to now. The main difference to the samples investigated in Refs. [8,9] is that in our case $l$ is smaller than $w$, i.e. the transport is diffusive also in the transverse direction, whereas $w \approx l / 2$ in Ref. [8]. This quasi-ballistic character of the samples in the transverse direction may cause the observed reduction of the rms-amplitude to $\delta G \approx 0.12 e^{2} / h[6$, $8,9]$.

The correlation field $B_{c}$ is given by the HWHM of the autocorrelation function of the magnetoconductance curves. It is obvious that $B_{c}$ of the curves shown in Fig. 1 decreases with increasing wire width. For ID samples with $w<\min \left(l_{\phi}, l_{\mathrm{T}}, l_{\mathrm{V}}\right)$ and $L \geqslant \min \left(l_{\phi}, l_{\mathrm{T}}, l_{\mathrm{V}}\right), B_{\mathrm{c}}$ should be 


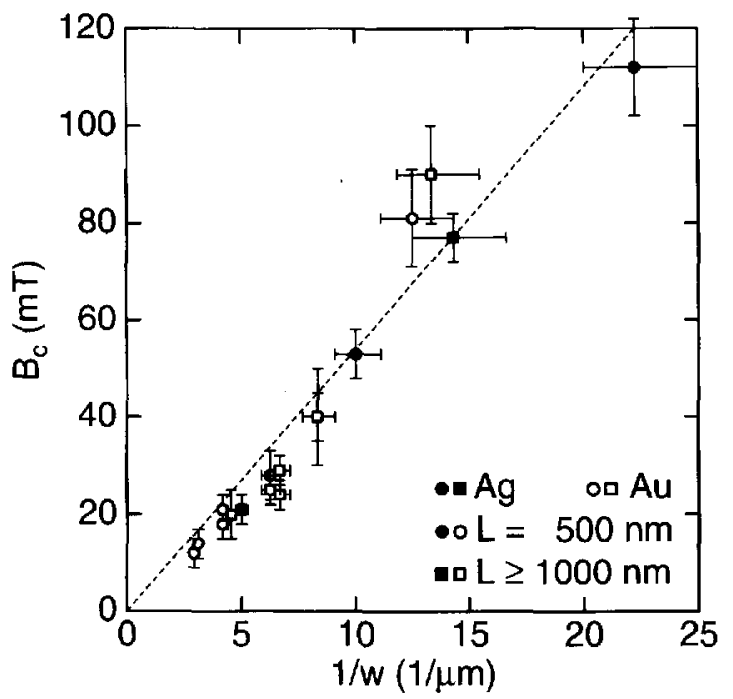

Fig. 2. Correlation field $B_{\mathrm{c}}$ versus inverse width $w^{-1}$ for gold and silver wires. The dashed line represents the expectation $B_{\mathrm{c}} \propto w^{-1}$.

inversely proportional to $w[3]$ :

$B_{\mathrm{c}}=C \frac{h}{e w l_{\phi}}$.

The expected behavior $B_{\mathrm{c}} \propto w^{-1}$ is roughly observed (cf. Fig. 2). The systematic deviations which occur for larger widths can be explained by minor contributions of the contact leads from the regions close to mesoscopic sample up to a depth of about $100 \mathrm{~nm}$ into each contact. This is in accordance with the slope of the dotted line, which gives $l_{\phi} \approx 700 \mathrm{~nm}$ for $C=1$. Within our accuracy we observe no systematic differences of $B_{\mathrm{c}}$ for gold and for silver samples.

We also investigated the correlation field of a narrow wire as a function of the angle $\theta$ between $B$ and current direction, i.e. varying the cross-section of the sample perpendicular to $B$. Fig. 3 shows $\delta G(B)$ of a Ag sample with $w=45 \mathrm{~nm}$ for five angles in the field range $-12.5 \mathrm{~T} \leqslant B \leqslant 12.5 \mathrm{~T}$. The accuracy of the angle adjustment is about $\pm 3^{\circ}$.

One observes a clear increase of the correlation field with decreasing $\theta$, but not only by damping of the high-frequency fluctuations, but also by appearance of new low-frequency ones. Therefore, the numerical evaluation of $B_{c}$ is not very stable and we have to perform a Fourier-filtering to cut-off the very long wavelengths $\Delta B>5 \mathrm{~T}$. These wavelengths correspond to areas smaller than $l^{2}$ and cannot therefore be evoked by quantum interference. The resulting $B_{\mathrm{c}}(\theta)$ is shown in Fig. 4, including the results for a second Ag sample with $w=100 \mathrm{~nm}$. Due to the Fourier filtering the values for $B_{\mathrm{c}}$ close to $\theta=0^{\circ}$ give only lower bounds. The solid

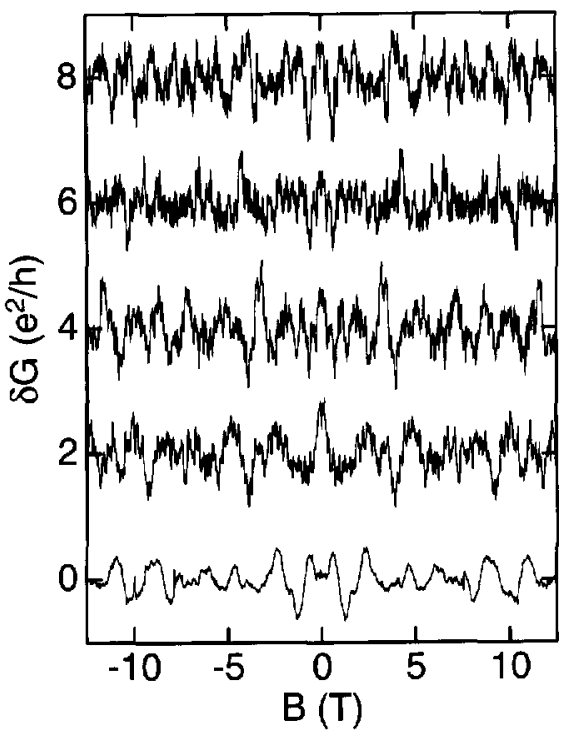

Fig. 3. Conductance fluctuations of an Ag sample with $w=45 \mathrm{~nm}$, $L=500 \mathrm{~nm}$, and $d=25 \mathrm{~nm}$ for various angles between magnetic field and current direction, offset by $2 e^{2} / h$ each. From top to bottom the angles are $90^{\circ}, 75^{\circ}, 60^{\circ}, 30^{\circ}$, and $0^{\circ}$.

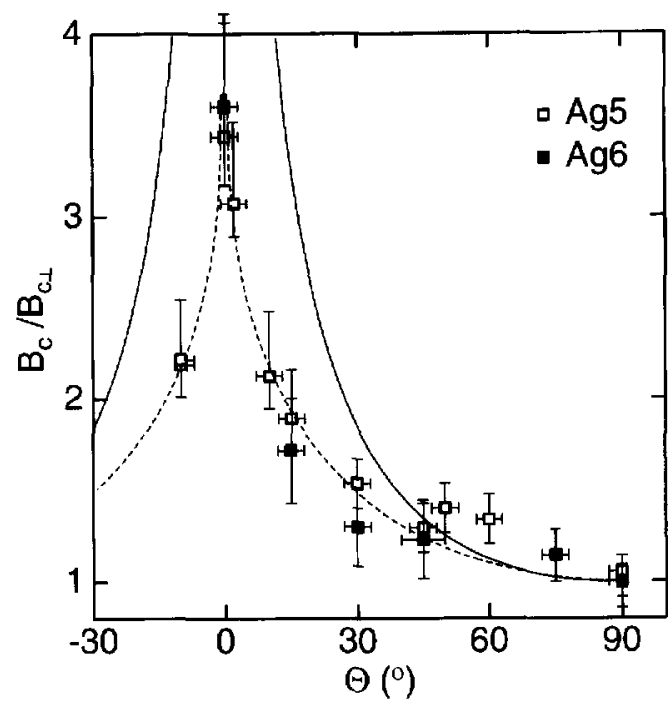

Fig. 4. Normalized correlation field $B_{\mathrm{c}} / B_{\mathrm{c} \perp}$ as a function of the angle $\theta$ between magnetic field and current direction for wires with $L=500 \mathrm{~nm}, d=25 \mathrm{~nm}$, and $w=45 \mathrm{~nm}$ (open symbols) and $w=100 \mathrm{~nm}$ (full symbols). The solid line represents the expected behavior (see text) and the dotted line is a guide to the eye.

line represents the expected angular dependence of $B_{\mathrm{c}}$ for the geometrical variation of the perpendicular cross-section taking into account the $3 \mathrm{D}$ shape of the sample. The functional dependence of the line is $B_{\mathrm{c}}=B_{\mathrm{c} \perp} / \sin \left(|\theta|+\theta_{0}\right)$, 
where $\theta_{0}=\arctan (d / L)$. This function approximates the geometrical dependence of $B_{\mathrm{c}}(\theta)$ for small $\theta_{0}$ and angles $|\theta| \leqslant 90^{\circ}-\theta_{0}$. For $\theta \leqslant 30^{\circ}$, a clear deviation between the prediction and the experimental result occurs, which cannot be explained by the mentioned Fourier filtering, influences of the contact leads or misalignment of the samples with respect to the magnetic field. Furthermore, the crosscorrelation of the fingerprints recorded at different angles is below significance. It is not possible to correlate the fluctuation patterns by scaling the field axis to the projection perpendicular to the sample area, as can be done for 2DEG samples [11]. A possible explanation of the difference is that for our samples surface scattering is the most important scattering process as is indicated by the appearance of quasiperiodic contributions to the fluctuations with a period which corresponds to the Altshuler-Aronov-Spivak period [12] $\Delta B=h /(2 e w d) \approx 2 \mathrm{~T}$ for the $45 \mathrm{~nm}$ sample. Therefore, the effective dimensionality of the sample changes from quasi1D to quasi-0D and the transport is not diffusive in both transverse directions. The averaging over many trajectories which is necessary for the validity of the ergodic hypothesis is not fulfilled any more. This means that the prefactor $C$ in Eq. (2) may not be the same for all angles [3, 13].

\section{Acknowledgements}

We would like to thank A. Sypli for her help in sample preparation. We are indebted to R. Schäfer, K. Hecker, and $\mathrm{H}$. Hegger for helpful discussions. This work was performed within the research program of Sonderforschungsbereich 195 supported by the Deutsche Forschungsgemeinschaft.

\section{References}

[1] C.P. Umbach, S. Washburn, R.B. Laibowitz and R.A. Webb, Phys. Rev. B 30 (1984) 4048.

[2] R.A. Webb, S. Washburn, C.P. Umbach and R.B. Laibowitz, Phys. Rev. Lett. 54 (1985) 2696.

[3] P.A. Lee and A.D. Stone, Phys. Rev. Lett. 55 (1985) 1622; P.A. Lee, A.D. Stone and H. Fukuyama, Phys. Rev. B 35 (1987) 1039

[4] D. Mailly, M. Sanquer, in: Quantum Coherence in Mesoscopic Systems, ed. B. Kramer (Plenum Press, New York, 1991) p. 401.

[5] A.B. Fowler, A. Hartstein and R.A. Webb, Phys. Rev. Lett. 48 (1982) 196.

[6] U. Murek, R. Schäfer and W. Langheinrich, Phys. Rev. Lett. 70 (1993) 841; P.A.M. Holweg, J. Caro, A.H. Verbruggen and S. Radelaar, Phys. Rev. B 48 (1993) 2479.

[7] P.A.M. Holweg, J.A. Kokkedee, J. Caro, A.H. Verbruggen, S. Radelaar, A.G.M. Jansen and P. Wyder, Phys. Rev. Lett. 67 (1991) 2549

[8] K. Hecker, H. Hegger, R. Schäfer, U. Murek, C. Braden and W. Langheinrich, Phys. Rev. B 50 (1994) 18601.

[9] V. Chandrasekhar and D.E. Prober, Phys. Rev. Lett. 61 (1988) 2253; V. Chandrasekhar, P. Santhanam and D.E. Prober, Phys. Rev. B 44 (1991) 11203

[10] E. Scheer, H. v. Löhneysen and H. Hein, J. Vac. Sci. Technol. B 12 (1994) 3171.

[11] S.B. Kaplan and A. Hartstein, Phys. Rev. Lett. 56 (1986) 2403; R.P. Taylor, M.L. Leadbeater, G.P. Whittington, P.C. Main, L. Eaves, S.P. Beaumont, I. McIntyre, S. Thoms and C.D.W. Wilkinson, Surf. Sci. 196 (1988) 52.

[12] B.L. Altshuler, A.G. Aronov, B.Z. Spivak, D.Yu. Sharvin and Yu.V. Sharvin, Sov. Phys. JETP Lett. 35 (1982) 588.

[13] C.W.J. Beenakker and H. van Houten, Phys. Rev. B 37 (1988) 6544. 